\title{
Efecto de una guía de intervención grupal en las habilidades sociales, en una muestra de adolescentes colombianos ${ }^{8}$
}

\author{
David Frost Cruz \\ Psicólogo \\ Universidad de La Sabana, Colombia \\ Correo electrónico: davidfrostcruz@gmail.com \\ Alejandra Madero Valle \\ Psicóloga \\ Universidad de La Sabana, Colombia \\ Correo electrónico: aleemadero9520@gmail.com
}

\section{Sthefany Maria Salomon Meza}

Psicóloga

Universidad de La Sabana, Colombia

Correo electrónico: sthefsame@gmail.com

\author{
Diana María Velasco Pinzón \\ Ms. en Psicología Clínica \\ Universidad de La Sabana, Colombia \\ Correo electrónico: diana.velasco11@gmail.com
}

Edgar Fernando Riveros Munevar
Ms. en Psicología
Universidad de La Sabana, Colombia Correo electrónico: edgar.riveros@unisabana.edu.co Autor de correspondencia

Carolina Méndez Sánchez

Ms. en Psicología Clínica

Universidad de La Sabana, Colombia

Correo electrónico: carolina.mendez2@unisabana.edu.co
Recibido: 26/06/2019

Evaluado: $15 / 08 / 2019$

Aceptado: 05/02/2020

\section{Resumen}

El objetivo del presente estudio fue determinar el efecto de la guía de intervención "Habilidades Sociales en Adolescentes, una Guía de Intervención Grupal diseñada para adolescentes de 12 a 17 años", en una institución educativa pública en el municipio de Chía, Colombia. La muestra de participantes estuvo conformada por 56 jóvenes, que fueron divididos en un grupo experimental a quienes se les aplicó la guía, y un grupo de comparación, quienes no recibieron ningún entrenamiento. Se realizó aplicación pre-test y pos-test de la Lista de Chequeo de Habilidades Sociales de Goldstein a ambos grupos con el fin de determinar los efectos de la guía. Los resultados arrojaron diferencias significativas en las medidas del grupo experimental, evidenciándose una mejora en las habilidades sociales en este grupo, mientras que en el grupo comparación no se evidenciaron cambios significativos, demostrando la eficacia de la guía, recomendando su replicación en otros contextos.

\section{Palabras clave}

Habilidades sociales, adolescencia, intervención grupal, protocolo de intervención.

8 Para citar este artículo: Frost, D., Madero, A., Salomon, S.M., Velasco, D.M., Riveros E.F., y Méndez, C. (2021). Efecto de una guía de intervención grupal en las habilidades sociales, en una muestra de adolescentes colombianos. Informes Psicológicos, 21(2), pp. 125-143 http://dx.doi.org/10.18566/infpsic.v21n2a08 


\section{Effect of a group intervention guide on social skills, in a sample of Colombian adolescents}

Abstract

The objective of this study was to determine the effect of the intervention guide "Social Skills in Adolescents, a Group Intervention Guide designed for adolescents between 12 and 17 years old", in a public educational institution in the municipality of Chía, Colombia. The sample of participants consisted of 56 young people, who were divided into an experimental group to whom the guide was applied, and a comparison group, who did not receive any training. A pre-test and post-test application of the Goldstein Social Skills Checklist was applied to both groups in order to determine the effects of the guide. The results showed significant differences in the measurements of the experimental group, showing an improvement in social skills in this group, while in the comparison group no significant changes were shown, demonstrating the effectiveness of the guide, and recommending its replication in other contexts.

\section{Efeito de um guia de intervenção em grupo nas habilidades sociais em uma amostra de adolescentes colombianos}

\section{Resumo}

0 objetivo deste estudo foi determinar 0 efeito do guia de intervenção "Habilidades Sociais em Adolescentes, um Guia de Intervenção em Grupo para adolescentes de 12 a 17 anos", em uma instituição de ensino pública no município de Chía, Colômbia. A amostra de participantes foi composta por 56 jovens, que foram divididos em um grupo experimental ao qual foi aplicado 0 guia e um grupo de comparação, que não recebeu nenhum treinamento. A aplicação do pré-teste e pós-teste da Lista de Habilidades Sociais de Goldstein foi aplicada a ambos os grupos para determinar os efeitos do guia. Os resultados mostraram diferenças significativas nas medidas do grupo experimental, evidenciando uma melhora nas habilidades sociais neste grupo, enquanto no grupo de comparação não foram apresentadas alterações significativas, demonstrando a eficácia do guia, recomendando sua replicação em outros contextos.

Palavras chave Habilidades sociais, adolescência, intervenção em grupo, protocolo de intervenção. 


\section{ntroducción}

En la población colombiana, las cifras de violencia interpersonal aumentaron de 64.803 (año 2017) a 66.218 (año 2018). De estas cifras, se registraron 1.911 casos de violencia interpersonal en personas entre 10 y 14 años de edad, así como 5.005 casos para las edades entre 15 y 19 años (Instituto Colombiano de Medicina Legal y Ciencias Forenses, 2018). De acuerdo con López de Mesa, Carvajal, Soto y Urrea (2013), los mayores índices de agresión interpersonal en jóvenes se evidencian en las aulas escolares. Estos incluyen el irrespeto por la autoridad, el rechazo a compañeros y otras agresiones de tipo verbal y físico que generan inadecuada convivencia escolar. Por lo anterior, se considera relevante la intervención en contextos escolares con el fin de influir en el desempeño social de los adolescentes, mejorando las relaciones interpersonales y facilitando el desarrollo de estrategias asertivas para la comunicación y resolución de problemas.

En este sentido, debe promoverse el establecimiento de relaciones sociales basadas en el respeto por sí mismo y el otro, la comunicación y la interacción a través del aprendizaje de habilidades sociales (HHSS), las cuales, además, permiten la prevención de conductas de riesgo durante la adolescencia, pues ejercen un rol fundamental en la construcción y funcionamiento eficaz de la sociedad (López de Mesa et al., 2013; Reyes, 2016). De esta manera, las habilidades sociales han sido estudiadas, abordadas y desarrolladas en diferentes y múltiples investigaciones, a través de las cuales se ha evidenciado que el entrenamiento y los programas de intervención en dichas habilidades demandan herramientas que se adapten a los diferentes contextos y prácticas actuales (Morán \& Olaz, 2014).

Según Caballo (1993), las habilidades sociales se pueden definir como un conjunto de conductas en las que tiene lugar la expresión asertiva de emociones, actitudes, opiniones y deseos de parte de un individuo en un espacio interpersonal, lo que implica respeto por el otro y una adecuada resolución de problemas. Por su parte, Goldstein, Sprafkin, Gershaw \& Klein (1989) señalan que las habilidades sociales se pueden clasificar dentro de 50 comportamientos específicos que se agrupan en seis componentes: 1) Habilidades sociales básicas, 2) Habilidades sociales avanzadas, 3) Habilidades relacionadas con los sentimientos, 4) Habilidades alternativas a la agresión, 5) Habilidades para hacer frente al estrés y 6) Habilidades de planificación.

La presente investigación está enfocada en adolescentes, ya que esta etapa del ciclo vital se considera crítica, en la medida en que representa un periodo de múltiples cambios físicos, psicológicos, cognitivos y sociales para el individuo (Patrício do Amaral, Maia \& Bezerra de Medeiros, 2015; Gur, 2015). Específicamente, a nivel psicológico y social, el adolescente genera un interés particular por la búsqueda de identidad, distanciándose probablemente de sus padres en el proceso de adquirir independencia, autonomía y lograr pertenencia a grupos de pares. Del mismo modo, en dicha transición, las 
personas cuentan con capacidades racionales para planificar su futuro, acercarse al establecimiento de creencias particulares sobre la vida, entender las emociones de los demás y preocuparse por el otro (Gaete, 2015).

Así mismo, existe evidencia empírica sobre la relación entre las habilidades sociales desarrolladas en la infancia y la adolescencia y el funcionamiento social, académico y psicológico en la adultez, confirmando así que los individuos con habilidades sociales adecuadas logran un mejor desempeño y adaptación en los ámbitos escolar, social y emocional, así como éxito en distintos aspectos de la vida (Albrecht, Mathur, Jones \& Alazemi, 2015). Además, esta etapa demanda repertorios conductuales más amplios y complejos en el relacionamiento con pares, así como el reconocimiento de los nuevos roles que deben cumplir los adolescentes en su proceso de desarrollo y en la sociedad a la que pertenecen, pues en gran medida el área social determina el funcionamiento en las relaciones sociales a futuro, influyendo en el bienestar psicológico (Holopainen, Lappalainen, Junttila \& Savolainen, 2012; Seema \& Kumar, 2018).

Por el contrario, la presencia de repertorios conductuales inefectivos o limitados en habilidades sociales suele estar relacionada con algunas problemáticas como conductas disruptivas o delincuencia en el contexto escolar, conductas de riesgo (autolesiones, ideación suicida, alcoholismo, etc.), baja tolerancia a la frustración y pronóstico complicado para psicopatologías como: esquizofrenia, ansiedad social, desórdenes alimenticios, trastornos de tipo emocional, etc. (Mendo, León del Barco, Castaño \& Polo del Río, 2016). Igualmente, un déficit en habilidades sociales puede asociarse con conductas externalizantes e internalizantes en los adolescentes, debido a la presencia de dificultades para comunicar sentimientos de forma apropiada, escaso conocimiento de las reglas sociales, dificultades para resolver problemas asertivamente y carencia de alternativas frente a la heteroagresión, entre otros (Albrecht et al., 2015).

Así pues, se entiende que el desarrollo adecuado de habilidades sociales en los adolescentes se encuentra ligado a la prevención de comportamientos y problemáticas como acoso escolar, sexismo, situaciones de violencia, dificultades en la manifestación de empatía, delincuencia juvenil y trastornos del estado de ánimo como la depresión (Garaigordobil \& Peña, 2014; Van Der Stouwe, Asscher, Hoeve, Van Der Laan \& Jan, 2018). De acuerdo con esto, las habilidades sociales constituyen gran parte del funcionamiento intragrupal $e$ intergrupal, al igual que influyen en el desarrollo del autoconcepto, la expresión de opiniones, la autonomía, la percepción sobre la aceptación de los demás y el soporte emocional, entre otros aspectos (Korem, Horenczyk \& Tatar, 2012).

En los últimos 10 años, se ha hecho evidente la influencia que ejercen las habilidades sociales en la adaptación general del individuo, el ajuste psicosocial y la vinculación hacia compromisos académicos y en otras áreas, considerándose un factor de protección y de prevención de comportamientos nocivos; por ejemplo, las investigaciones 
demuestran que individuos con dificultades para expresar negativas, son víctimas con mayor frecuencia e intensidad de agresiones, amenazas e intimidación, mientras que el establecimiento de habilidades sociales adecuadas se convierte en un indicador de desarrollo psicológico saludable y un predictor de buen rendimiento académico (Bautista, Plata, Hernández, Herrera \& Parra, 2017; Garaigordobil \& Peña, 2014; Wolstencroft et al., 2018). Por lo tanto, los hallazgos presentes en diversos estudios han impulsado el desarrollo de variedad de técnicas y modalidades de intervención en el tema, entre las que se encuentra el entrenamiento en habilidades sociales, dirigido a favorecer conductas prosociales y a prevenir o modificar comportamientos que generen dificultades interpersonales en los adolescentes (Garaigordobil \& Peña, 2014).

De acuerdo con lo anterior, diferentes investigaciones demuestran la efectividad de la intervención en habilidades sociales a nivel grupal (Li et al., 2016; Van Vugt, Deković, Prinzie, Stams \& Asscher, 2013). Además, entre las técnicas más utilizadas para cumplir con este propósito se encuentran el modelamiento, el moldeamiento, la reestructuración cognitiva y el reforzamiento positivo de las habilidades específicas; dichas estrategias hacen parte del enfoque Cognitivo-Conductual, el cual demuestra mayor evidencia empírica para la intervención en este tipo de problemáticas (Gadaire, Marshall \& Brissett, 2017; González, Ampudia \& Guevara, 2012; Li et al., 2016; McDaniel, Bruhn \& Troughton, 2017; Probst, Geib, Güroff \& Mühlberger, 2017). También es común que se incluyan componentes como la explicación de las habilidades sociales, ejemplos acordes a los contextos de interacción, juego de roles, ensayos conductuales y la asignación de ejercicios para practicar las habilidades por fuera del escenario de intervención (Van Vugt et al., 2013; Li et al., 2016; McDaniel et al., 2017).

Ahora bien, es habitual que este tipo de intervenciones se realicen de forma grupal y se cuente con homogeneidad en el grupo, en aspectos relevantes como la edad, esto con el fin de prevenir que existan diferencias muy amplias que puedan conllevar a un entorno disfuncional en el grupo (Sanz, 2016). Por otro lado, se suele agrupar a los participantes de acuerdo a características psicopatológicas comunes, tales como Trastorno por Déficit de Atención e Hiperactividad (TDAH), Autismo, Asperger y dificultades de aprendizaje, entre otras; de hecho, la literatura expone numerosas investigaciones con las posibles psicopatologías mencionadas (García-Castellar, Jara-Jiménez, Sánchez-Chiva \& Mikami, 2015; Mafra, 2015; Radley, Jenson, Clark, Hood \& Nicholas, 2014).

Del mismo modo, es frecuente que las investigaciones relacionadas con el entrenamiento de habilidades sociales a niños y adolescentes ocurran al interior del contexto escolar, ya que es un contexto adecuado para la observación y práctica de las estrategias, demostrando resultados positivos en las intervenciones en cuanto al aumento de los repertorios adecuados (Caballo, CarriIlo \& Ollendick, 2015; Vagos, Pereira \& Warner, 2015). Algunas investigaciones demuestran, incluso, que las intervenciones grupales en habilidades sociales 
con enfoque Cognitivo-Conductual, contribuyen al aumento de los niveles de autoeficacia y favorecen la interacción social en los adolescentes (Caballo et al., 2015). Otros estudios confirman aspectos interesantes del desarrollo por parte de los adolescentes, de los niveles de comprensión sobre los pensamientos relacionados con aspectos sociales, simultáneo a que refieren un importante aumento en las conductas prosociales, debido al trabajo en temas como autoestima, responsabilidad, valores y metas a futuro, toma de decisiones y manejo adecuado de repertorios emocionales, entre otros (Harrell, Mercer \& DeRosier, 2009). Finalmente, algunas investigaciones indican que cuando los adolescentes cuentan con niveles elevados de empatía, las intervenciones grupales en habilidades sociales pueden aportar a la disminución de conductas hostiles o agresivas (Van der Stouwe et al., 2018). Derivado de lo anterior, el presente estudio se propone como objetivo determinar el efecto de la guía de intervención: "Habilidades Sociales en Adolescentes, una Guía de Intervención Grupal diseñada para adolescentes de 12 a 17 años", en una institución educativa pública en el municipio de Chía, Colombia.

Es relevante tener en cuenta que esta guía fue diseñada en un centro de servicios psicológicos que tiene su zona de influencia en la Sabana centro de Cundinamarca. Para fines de la presente investigación, es relevante considerar que ésta es la primera vez que se aplica la guía, y el primer proceso de validación que se realiza con este instrumento.

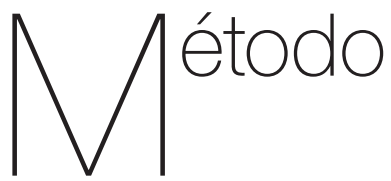

\section{Diseño}

La presente investigación tuvo un enfoque cuantitativo desde el paradigma empírico analítico experimental, con un diseño cuasiexperimental de tipo pre-test y pos-test, con grupo de comparación bajo regla de asignación conocida para la selección de los participantes. Se considera cuasiexperimental debido a que no se realizó un proceso de aleatorización para la selección de la muestra, sino que se escogieron datos en grupos ya conformados. En este tipo de estudio se pretende manipular una variable independiente para observar el efecto en un conjunto de variables dependientes, y difiere del modelo experimental en el grado de confiabilidad que pueda haber sobre la equivalencia inicial de los grupos (Hernández, Fernández \& Baptista, 2014). En este caso específico, se eligieron participantes pertenecientes a una institución educativa del municipio de Chía, Colombia, siendo la muestra experimental constituida por los estudiantes del grado noveno: uno de los cursos como muestra experimental, y otro de los cursos como grupo de comparación.

\section{Participantes}

La muestra estuvo compuesta por un total de 56 participantes, cuya asignación se realizó mediante un muestreo no probabilístico intencional, y los criterios de inclusión sólo consistían en pertenecer a la institución educativa particular, estar en grado noveno y 
encontrarse en un rango de edad entre los 12 y 17 años. Es necesario tener en cuenta que el muestreo no probabilístico depende del proceso de toma de decisiones de los investigadores, y responde a los criterios de inclusión que son planteados desde el ejercicio investigativo, al igual que no se pretende que los casos sean representativos para la población (Hernández et al., 2014). grupo experimental correspondió a un total de 27 participantes, de los cuales 13 fueron hombres y 14 mujeres, pertenecientes a estratos socioeconómi$\cos 1,2$ y 3 . Por su parte, el grupo de comparación estuvo compuesto por 29 participantes, 11 mujeres y 18 hombres, pertenecientes también a estratos socioeconómicos 1, 2 y 3. Respecto a la estructura familiar, el $51.8 \%$ de los participantes pertenece a una familia extensa, el $35.7 \%$ del total de los participantes pertenece a una familia nuclear, el $7.1 \%$ a una familia monoparental femenina, el $3.6 \%$ a una familia recompuesta, y el $1.8 \%$ a una familia recompuesta-extensa. Finalmente, de la muestra total, 7 participantes (4 del grupo experimental y 3 del grupo control) habían consultado anteriormente con algún especialista en salud mental: psiquiatría, psicología, neuropsicología, etc.

\section{Instrumentos}

\section{Guía de intervención grupal.}

La guía se compone de trece sesiones en las que se trabajan las habilidades planteadas por Goldstein et al. (1989); incluye para el entrenamiento técnicas como modelamiento y moldeamiento de las habilidades, además de estrategias para facilitar el aprendizaje como juego de roles, asignación de tareas, materiales interactivos, recursos audiovisuales y actividades lúdicas. Inicialmente, la guía fue diseñada para intervenciones grupales en psicología clínica y tiene por objetivo proponer estrategias de intervención psicológica para la educación y el entrenamiento en habilidades sociales para adolescentes (entre 12 y 17 años), con el fin de facilitar la adquisición y puesta en práctica de herramientas que contribuyan a un desarrollo apropiado y acorde a su etapa del ciclo vital (Díaz, Díaz \& Frost, 2018). Se considera necesario aclarar que, aun cuando la guía fue diseñada para el ámbito clínico, puede ser aplicada en población no clínica, puesto que frecuentemente investigaciones sobre el tema de habilidades sociales tienen lugar en contextos diferentes al de psicología clínica, entre estos contextos resulta principal el escolar, debido al fácil acceso a la población de adolescentes (Vagos et al., 2015).

La aplicación de las sesiones tuvo una frecuencia de dos veces por semana al interior del colegio, tanto en los salones de clase como en otras instalaciones que provistas por el colegio. Los facilitadores en cada sesión fueron tres profesionales en formación del Centro de Servicios de Psicología de la Clínica Universidad de La Sabana, quienes recibieron acompañamiento y supervisión de parte de equipo de profesionales en psicología clínica del Centro de Servicios de Psicología. A continuación, se presentan los objetivos y los temas a abordar por sesiones. 


\section{Tabla 1.}

Habilidades Sociales en Adolescentes: Una Guía de Intervención Grupal (Díaz et al., 2018).

\begin{tabular}{|c|c|c|}
\hline & Sesión & Objetivo de la sesión \\
\hline 1 & $\begin{array}{l}\text { Encuadre } \\
\text { y espacio } \\
\text { de relación } \\
\text { colaborativa. * }\end{array}$ & $\begin{array}{l}\text { Generar un espacio en que los participantes conozcan a los terapeutas, y generar una relación } \\
\text { colaborativa. Se inicia con la explicación del protocolo dando cuenta de sus dinámicas, alcances } \\
\text { y objetivos y una actividad para favorecer la relación colaborativa. Asimismo, se realiza la aplica- } \\
\text { ción de la evaluación pre-test y se establecen las normas y reglas del grupo en conjunto con los } \\
\text { participantes. }\end{array}$ \\
\hline 2 & $\begin{array}{c}\text { Habilidades } \\
\text { sociales básicas I.* }\end{array}$ & $\begin{array}{l}\text { Entrenar y practicar habilidades sociales básicas: escuchar, iniciar una conversación y mantenerla. } \\
\text { Se inicia con la explicación del objetivo. A través de un video y de una explicación verbal, los tera- } \\
\text { peutas dan inicio a explicación de las habilidades sociales básicas pidiéndoles identificar si se rea- } \\
\text { lizan de forma adecuada o no. Se realiza una actividad lúdica diseñada para apliquen habilidades } \\
\text { sociales desde la utilización del lenguaje no verbal. Los terapeutas repasan las habilidades. Para } \\
\text { consolidar la información, los participantes hacen un juego de roles, el cual finaliza retroalimenta- } \\
\text { cion sobre los temas abordados. }\end{array}$ \\
\hline 3 & $\begin{array}{l}\text { Habilidades } \\
\text { sociales } \\
\text { básicas II.* }\end{array}$ & $\begin{array}{l}\text { Entrenar y practicar habilidades sociales básicas: preguntar, agradecer, presentarse, presentar } \\
\text { a otro y halagar. Se inicia explicando cada habilidad, y se realiza modelamiento por parte de los } \\
\text { terapeutas. Posteriormente se explica cada habilidad a través de unos pasos que se determinan } \\
\text { en la guía. Para consolidar la información, los participantes realizan una actividad individual para } \\
\text { poner en prácticas las habilidades con ellos mismos y un juego de roles que evidencien el apren- } \\
\text { dizaje de cada una de las habilidades abordadas entre las que se contemplan las mencionadas } \\
\text { anteriormente. }\end{array}$ \\
\hline 4 & $\begin{array}{l}\text { Habilidades } \\
\text { sociales } \\
\text { avanzadas.* }\end{array}$ & $\begin{array}{l}\text { Entrenar y practicar habilidades sociales avanzadas: pedir ayuda, dar y seguir instrucciones, dis- } \\
\text { culparse y convencer a los demás. Se inicia con una actividad rompehielo y se presenta el objetivo } \\
\text { de la sesión. Se continúa con una explicación de las habilidades y un juego de roles posterior sobre } \\
\text { dos situaciones que reúnan las últimas habilidades, las cuales impliquen la puesta en práctica de } \\
\text { las demás. Se realiza retroalimentación de la sesión. }\end{array}$ \\
\hline 5 & $\begin{array}{l}\text { Habilidades } \\
\text { relacionadas con } \\
\text { sentimientos I.* }\end{array}$ & $\begin{array}{l}\text { Entrenar y practicar habilidades relacionadas con los sentimientos: conocer los propios sentimien- } \\
\text { tos, expresar los sentimientos y comprender los sentimientos de los demás. Se realiza una activi- } \\
\text { dad rompehielo que consiste en reunir expresión de emociones, junto a los pensamientos y mostrar } \\
\text { su asociación ante situaciones desencadenantes. Posteriormente, se lleva a cabo otra actividad en } \\
\text { la que se incluye una metáfora, explicando a los participantes que las emociones son fenómenos } \\
\text { psicológicos adaptativos que acompañan todas las experiencias de la vida. En esta actividad se les } \\
\text { pide a los consultantes identificar situaciones de su vida personal donde se evoque cierta emoción } \\
\text { específica y se compartan con el grupo. Se les solicita que representen esta situación identificando } \\
\text { las sensaciones fisiológicas y pensamientos relacionados con dicha emoción. Igualmente, se les } \\
\text { recuerda la importancia de evitar juzgar la experiencia emocional. A seguir, se ejecuta en conjunto } \\
\text { la expresión de dichas situaciones buscando favorecer el proceso de validación emocional. Se } \\
\text { retroalimenta al finalizar la actividad y se asigna tarea para iniciar la temática de la siguiente sesión } \\
\text { sugiriendo realizar la aplicación durante la semana siguiente. }\end{array}$ \\
\hline 6 & $\begin{array}{l}\text { Habilidades } \\
\text { relacionadas con } \\
\text { sentimientos Il.* }\end{array}$ & $\begin{array}{l}\text { Entrenar y practicar habilidades relacionadas con los sentimientos: expresar afecto, resolver el } \\
\text { miedo y auto recompensarse. Se presenta el objetivo de la sesión, se indaga sobre la tarea asig- } \\
\text { nada y se retoma la actividad dejada como tarea en la anterior sesión, mostrando las imágenes } \\
\text { relacionadas con la expresión del efecto en el que se recogen las impresiones sobre lo que ocurre } \\
\text { en dichas imágenes. Se explican las habilidades relacionadas con expresar afecto. En cuanto a la } \\
\text { habilidad de resolver el miedo, se destaca la diferencia entre situaciones poco probables y cotidia- } \\
\text { nas, se realiza ensayo conductual. En cuanto a la habilidad de autorecompensarse, se dan algunos } \\
\text { ejemplos sobre cómo realizar la aplicación de dicha habilidad. Se retroalimenta la sesión. }\end{array}$ \\
\hline 7 & $\begin{array}{l}\text { Habilidades } \\
\text { alternativas a } \\
\text { la agresión I.* }\end{array}$ & $\begin{array}{l}\text { Entrenar y practicar habilidades alternativas a las conductas agresivas: pedir permiso, compartir } \\
\text { algo, ayudar a los demás, negociar y tener autocontrol. Se presenta el objetivo de la sesión, reali- } \\
\text { zando actividades de observar fragmentos de películas e identificar la agresión y la solución. Luego } \\
\text { se procede a la explicación de cada una de las habilidades y se finaliza con un juego de roles que } \\
\text { consolide la información. Se retroalimenta la sesión. }\end{array}$ \\
\hline
\end{tabular}




\begin{tabular}{|c|c|c|}
\hline & Sesión & Objetivo de la sesión \\
\hline 8 & $\begin{array}{l}\text { Habilidades } \\
\text { alternativas a la } \\
\text { agresión II. }{ }^{*}\end{array}$ & $\begin{array}{l}\text { Entrenar y practicar habilidades alternativas a las conductas agresivas: defender los propios dere- } \\
\text { chos, responder a las bromas, evitar problemas con los demás y no entrar en peleas. Se presenta } \\
\text { el objetivo de la sesión, se explican las habilidades a desarrollar durante la misma iniciando po } \\
\text { defender los propios derechos, y se recrean situaciones cotidianas que posiblemente ocurren en e } \\
\text { contexto escolar llevando a cabo un juego de roles buscando favorecer la habilidad inicial. Poste-- } \\
\text { riormente, se explica la habilidad de responder a las bromas; en este caso, se recrean dos situa- } \\
\text { ciones en las que, por un lado, se presenta un chiste y, por otro, comentarios groseros e insultos } \\
\text { Se ejecuta un juego de roles con las habilidades adquiridas únicamente con dos consultantes que } \\
\text { simulan la situación. Para las habilidades de evitar problemas con los demás y no entrar en peleas } \\
\text { se presentan unas imágenes con la finalidad de encontrar alternativas de solución a las mismas } \\
\text { Se realiza explicación de las habilidades y se replantean las alternativas de solución buscando que } \\
\text { éstas logren ser asertivas. Se retroalimenta la sesión. }\end{array}$ \\
\hline
\end{tabular}

Entrenar y practicar habilidades alternativas a las conductas agresivas: defender los propios deredefender los propios derechos, y se recrean situaciones cotidianas que posiblemente ocurren en el contexto escolar llevando a cabo un juego de roles buscando favorecer la habilidad inicial. Posteliormente, se explica la habilidad de responder a las bromas; en este caso, se recrean dos situaciones en las que, por un lado, se presenta un chiste y, por otro, comentarios groseros e insultos. Se ejecuta un juego de roles con las habilidades adquiridas únicamente con dos consultantes que (a) Se realiza explicación de las habilidades y se replantean las alternativas de solución buscando que ex ser asertivas. Se retroalimenta la

Habilidades para hacer frente al estrés I.*
Habilidades para hacer frente al estrés II.*
Entrenar y practicar habilidades para hacer frente al estrés: formular una queja, responder a una queja, resolver la vergüenza, arreglárselas cuando le dejan de lado y defender a un amigo. Se realiza una actividad rompehielo y se presenta el objetivo de la sesión. Se explican las primeras dos habilidades y se simulan dos situaciones consignadas en la guía para realizar un juego de roles en las que éstas puedan ser aplicadas; en el desarrollo de estas habilidades, se pide que sus compañeros sean diferentes en ambas situaciones. Después, se explica la habilidad de la vergüenza y se simulan otras dos situaciones sobre las cuales se realiza un juego de roles. Para la habilidad de arreglárselas cuando le dejan de lado, se explica la habilidad y se simulan otras dos situaciones contempladas en la guía sobre las que se realizan juego de roles. En cuanto a la habilidad de defender a un amigo, se realiza el mismo procedimiento de las demás habilidades. Se retroalimenta la sesión.

Entrenar y practicar habilidades para hacer frente al estrés: responder a la persuasión, responder al fracaso, enfrentarse a los mensajes contradictorios, responder a una acusación, prepararse para una conversación difícil y hacer frente a las presiones del grupo. Se inicia con una actividad rompehielo y se presenta el objetivo. Después, se realiza una actividad lúdica en la que se simula una situación donde los consultantes se encuentran en un juzgado buscando generar la aplicación adecuada de las habilidades. Se retroalimenta la sesión con la explicación de la forma adecuada de aplicación de las habilidades.

\begin{tabular}{|c|c|c|}
\hline 11 & $\begin{array}{l}\text { Habilidades de } \\
\text { planificación I.* }\end{array}$ & $\begin{array}{l}\text { Entrenar y practicar habilidades de planificación: tomar iniciativa, discernir sobre la causa de un } \\
\text { problema, establecer un objetivo y determinar las propias habilidades. Se realiza una actividad } \\
\text { rompehielo y se presenta el objetivo de la sesión. Para cada una de las habilidades, se sigue el } \\
\text { siguiente procedimiento: se explica la habilidad y se realiza un ejercicio experiencial en el que se } \\
\text { le pide identificar situaciones cotidianas sobre las cuales es recomendable abordar las estrategias } \\
\text { planteadas. Al finalizar, para la habilidad de determinar las propias habilidades, se tienen en cuenta } \\
\text { las demás habilidades y se les solicita considerarlas en otro ejercicio experiencial. }\end{array}$ \\
\hline
\end{tabular}

Entrenar y practicar habilidades de planificación: recoger información, resolver los problemas según su importancia, tomar una decisión y concentrarse en una tarea. Se realiza una actividad $\begin{array}{ll}\text { Habilidades de } & \text { rompehielo, presentación del objetivo y se simula una actividad lúdica en la que se representa una } \\ \text { situación problemática a resolver. En esta actividad, se tienen en cuenta situaciones que pueden }\end{array}$ planificación II. $\quad$ ocurrir en la cotidianidad de los participantes adolescentes. Se busca que logren derivar el proceso de planificación teniendo en cuenta cada una de las habilidades abordadas. Se retroalimenta la sesión.

\begin{tabular}{|c|c|c|}
\hline 12 & $\begin{array}{l}\text { Habilidades de } \\
\text { planificación II.* }\end{array}$ & $\begin{array}{l}\text { rompehielo, presentación del objetivo y se simula una actividad lúdica en la que se representa una } \\
\text { situación problemática a resolver. En esta actividad, se tienen en cuenta situaciones que pueden } \\
\text { ocurrir en la cotidianidad de los participantes adolescentes. Se busca que logren derivar el proceso } \\
\text { de planificación teniendo en cuenta cada una de las habilidades abordadas. Se retroalimenta la } \\
\text { sesión. }\end{array}$ \\
\hline 13 & $\begin{array}{l}\text { Cierre del } \\
\text { proceso." }\end{array}$ & $\begin{array}{l}\text { Realizar el cierre de la terapia grupal mediante una actividad lúdica. Aplicar la evaluación pos- } \\
\text { test. Se inicia con la aplicación del instrumento para medida pos-test y una actividad rompehie- } \\
\text { lo. Posteriormente, se retroalimenta el proceso y se favorece el proceso de autoeficacia con los } \\
\text { participantes. }\end{array}$ \\
\hline
\end{tabular}

\section{Fuente: elaboración propia.}

Nota: *Se considera útil tener en cuenta que, en cada una de las sesiones, los consultantes deben diligenciar un documento contemplado en un cuadernillo en el que se entrega el resumen de las habilidades abordadas. En este documento, se recogen impresiones acerca del aprendizaje generado durante la sesión y se retoman al iniciar cada una de las sesiones. Igualmente, la guía indica que cuando se explican habilidades se retomen experiencias de la vida personal de los consultantes buscando favorecer el proceso de aprendizaje de los repertorios conductuales adecuados. 
Lista de Chequeo de Habilidades Sociales de Goldstein et al. (1989).

Esta lista de chequeo evalúa las capacidades respecto al uso de las habilidades sociales propuestas por Goldstein et al. (1989). Se compone de 50 ítems repartidos en 6 dimensiones: 1) Habilidades sociales básicas, 2) Habilidades sociales avanzadas, 3) Habilidades relacionadas con los sentimientos, 4) Habilidades alternativas a la agresión, 5) Habilidades para hacer frente al estrés y 6) Habilidades de planificación. Consiste en una escala de tipo Likert en la que se puntúa cada ítem de 1 a 5 y los valores de puntuación equivalen a: 1 = nunca se presenta la situación, $2=$ se presenta pocas veces, $3=$ se presenta a veces, $4=$ se presenta a menudo y 5 = se presenta siempre. No se cuentan con datos para población colombiana, sin embargo, derivado de la aplicación del instrumento para esta investigación se logró obtener un alfa de Cronbach = .920, lo que indica una confiabilidad alta.

\section{Procedimiento}

Se realizó el proceso de selección de la institución educativa, la cual autorizó su participación en la investigación. A seguir, se presentó y firmó el consentimiento informado que incluye el objetivo de la investigación, los alcances, la metodología y la confidencialidad de los datos sobre la identidad de los participantes; este proceso se realizó con la autorización de los padres de cada uno de los participantes (menores de edad).

A continuación, se procedió a la aplicación de la guía por medio del desarrollo de cada una de las sesiones. Al finalizar el proceso, se aplicó nuevamente la Lista de Chequeo de Habilidades Sociales con el fin de completar la fase de evaluación postest para ambas muestras (Goldstein et al., 1989). Posteriormente, se efectuó el análisis de resultados, en el que se tuvieron en cuenta los puntajes obtenidos en el instrumento para ambos grupos. Finalmente, se tenía planeado realizar la aplicación con el grupo de comparación para el inicio del año 2020.

\section{Consideraciones éticas}

Se contó con las especificaciones de confidencialidad de los datos y de las condiciones para la realización de investigaciones, expresadas en el código 52 de la Ley 1090 del año 2006, en el Código Deontológico y Bioético del Ejercicio de la Psicología en Colombia; dichas especificaciones responden al principio ético del respeto y la dignidad de los participantes, así como al aseguramiento de su bienestar y sus derechos. Cabe mencionar que, al finalizar el estudio, se planteó la aplicación al grupo de comparación como parte del proceso ético recomendable para este tipo de intervenciones. Igualmente, no se presenta conflicto de intereses por parte de los investigadores.

\section{Análisis de resultados}

Se consolidaron los resultados para realizar los análisis estadísticos pertinentes, específicamente la prueba de ajuste Shapiro-Wilk (SW), la prueba de U de Mann Whitney, t de Student para muestras emparejadas y prueba de Wilcoxon, que posibilitaron la consecución del objetivo 
principal: determinar el efecto de la guía de intervención. Estos estadísticos se propusieron con la finalidad de verificar la normalidad en la distribución de los datos, utilizando específicamente la prueba de SW, dado el tamaño de la muestra debido que esta prueba se emplea cuando el n es menor a 30 (Pedrosa, Juarros-Basterretxea, Robles-Fernández, Basteiro \& García-Cueto, 2015); y de establecer pruebas no paramétricas sobre los datos de las muestras independientes que forman parte de la investigación, en este caso, la prueba $U$ de Mann-Whitney para muestras independientes, así como la medida de la prueba de Wilcoxon y la prueba paramétrica de t de Student para muestras emparejadas (Rodríguez, Gutiérrez \& Pozo, 2014). Finalmente, se generaron las conclusiones de la investigación, se retroalimentaron los resultados a los participantes y se entregaron las recomendaciones generales a la institución educativa.

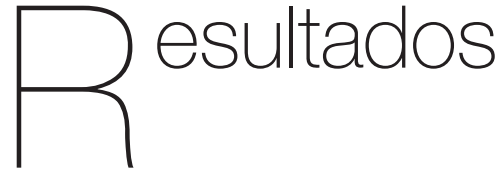

\section{Análisis de la distribución de normalidad de los datos en las medidas pre-test y pos-test de ambos grupos.}

A continuación, se presentan los resultados de la prueba de normalidad para cada uno de los puntajes arrojados, tanto para el grupo experimental como para el grupo de comparación.

Tabla 2.

Resultados de la prueba Shapiro-Wilk para los datos iniciales en las medidas pre-test y pos-test del grupo experimental y de comparación.

\begin{tabular}{lccc}
\hline & Grupo $^{*}$ & Estadístico & Sig. \\
\hline Experimental & Total pre-test & .900 & .013 \\
& Total pos-test & .977 & .801 \\
\cline { 2 - 4 } Comparación & Total pre-test & .965 & .433 \\
& Total pos-test & .958 & .291 \\
\hline
\end{tabular}

Nota: *Es necesario tener en cuenta que la prueba Shapiro-Wilk se emplea para muestras que corresponden a un número menor a 30 participantes. Por ello, se utilizó esta prueba de normalidad, donde una significancia estadística mayor al Alfa .05, permite asumir distribución normal en los datos.

En la Tabla 2 se puede observar que la significancia SW del grupo experimental en el pre-test fue menor al Alfa .05, por lo que se asume una distribución no normal de los datos, lo que refiere un análisis no paramétrico de los resultados relacionados con la evaluación pre-test del grupo experimental. Por otra parte, la significancia SW de la evaluación pos-test en el grupo experimental supera el Alfa de .05, aspecto que indica normalidad para dichos datos implicando un análisis paramétrico. En este sentido, también se sugiere un análisis paramétrico para el grupo de comparación en ambas medidas (pre-test y pos-test), puesto que se obtiene una significancia mayor al Alfa. 


\section{Análisis descriptivo de los puntajes en ambas medidas (pre- test y pos-test) para los dos grupos.}

En la Tabla 3 se reúnen los datos descriptivos que dan cuenta de la puntuación media de cada uno de los grupos en ambos momentos de la evaluación, así como también recoge la desviación estándar en los puntajes obtenidos.

Tabla 3.

Estadísticos descriptivos de los resultados obtenidos en el instrumento.

\begin{tabular}{cccc}
\hline & Grupo & Resultados pre-test & Resultados pos-test \\
\hline Experimental & Media & 169.26 & 177.77 \\
& Desviación estándar & 28.85 & 25.71 \\
\cline { 2 - 4 } Comparación & Media & 169.97 & 170 \\
& Desviación estándar & 25.3 & 23.48 \\
\hline
\end{tabular}

De acuerdo con la Tabla 3, se puede observar que la media de los puntajes del grupo experimental tuvo un cambio importante en la evaluación pos-test, mientras que los resultados de la aplicación del instrumento en el grupo de comparación muestran similitud, pues difieren en pocas unidades.

\section{Análisis de pruebas} no paramétricas para las muestras independientes en las puntuaciones pre-test.

Como en las medidas anteriores del grupo experimental, en la fase pretest no se obtuvo normalidad en los datos, y se empleó un análisis estadístico no paramétrico: la prueba de $U$ de Mann-Whitney.

Tabla 4.

Resultados estadísticos de la prueba $U$ de MannWhitney para la fase pre-test entre los grupos.

\begin{tabular}{cc}
\hline & Puntaje pre-test \\
\hline U de Mann-Whitney & 384.5
\end{tabular}

Sig. asintótica (bilateral)

.909

Los resultados de la Tabla 4 dan cuenta de una significancia superior al .05, lo que permite afirmar que no existen diferencias estadísticamente significativas en las puntuaciones pre-test, entre los grupos experimental y de comparación, de allí que se garantice una equivalencia inicial de los grupos. 


\section{Análisis de pruebas} paramétricas para las muestras independientes en las puntuaciones pre-test y pos-test de ambos
grupos.

Con el fin de determinar si existieron cambios estadísticamente significativos al interior de los grupos, se realizó un análisis de diferencia de medidas con la prueba t de Student para el grupo de comparación y con la prueba de Wilcoxon para el grupo experimental.

Tabla 5.

Resultados de prueba t para muestras emparejadas.

\begin{tabular}{ccc}
\hline & Grupo & Sig. (bilateral) \\
\hline Comparación & Puntuación pre-test pos-test & .992 \\
\hline
\end{tabular}

Como puede observarse en la Tabla 5, para el grupo de comparación se encuentra una significancia superior al .05, indicando que no existieron cambios estadísticamente significativos entre la pre prueba y la post prueba.

Tabla 6.

Estadísticos de prueba de rangos con signo de Wilcoxon

\begin{tabular}{ccc}
\hline & Grupo & Puntajes pos-test y pos-test \\
\hline Experimental & $\mathrm{Z}$ & -2.49 \\
\cline { 2 - 3 } & Sig. asintótica (bilateral) & .013 \\
\hline
\end{tabular}

Los datos de la prueba de Wilcoxon (ver Tabla 6) permiten dar cuenta de una significancia inferior al .05, indicando diferencias estadísticamente significativas a favor del pos- test, lo que refiere que la variable del tratamiento generó mejoría estadística en las habilidades sociales de los participantes del grupo experimental.

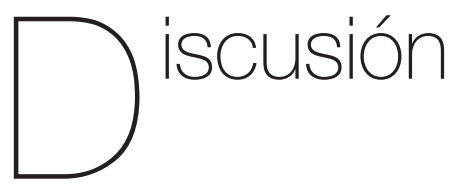

La presente investigación se propuso como objetivo determinar el efecto de la guía de intervención: "Habilidades Sociales en Adolescentes, una Guía de Intervención Grupal diseñada para adolescentes de 12 a 17 años", en una institución educativa pública en el municipio de Chía, Colombia. De acuerdo con lo anterior, los datos pre-test evidencian equivalencia en ambos grupos, lo que significa que la media de los datos obtenidos es proporcional para los grupos y no presenta diferencias significativas. Las medidas pos-test demuestran que hubo cambios positivos en las habilidades sociales del grupo experimental. En otra medida, los datos del pre-test y el pos-test del grupo de comparación no muestran diferencias 
estadísticamente significativas, razón por la cual se podría establecer que la variable independiente (guía de intervención), tuvo un efecto significativo en las habilidades sociales de los participantes del grupo experimental, demostrado a partir de las mejoras en la puntuación del instrumento. Lo anterior resulta consistente con lo planteado por Caballo et al. (2015), quienes argumentan que las intervenciones grupales de habilidades sociales desde un enfoque Cognitivo-Conductual influyen positivamente la interacción social en los adolescentes.

Del mismo modo, los resultados permiten corroborar en cierta medida aspectos importantes de las investigaciones realizadas sobre este tema. En consecuencia, se logra demostrar que, así como lo proponen Li et al. (2016) y Van Vugt et al. (2013), las intervenciones en habilidades sociales a nivel grupal demuestran efectividad para el desarrollo de dichas habilidades de forma adecuada. De igual manera, la utilización de técnicas y estrategias del enfoque Cognitivo-Conductual como el modelamiento, el moldeamiento, los juegos de roles y el refuerzo positivo, entre otras, resultan efectivas en términos de la intervención realizada (Gadaire et al., 2017; Li et al., 2016; McDaniel et al., 2017). Lo anterior se puede establecer a partir de los resultados obtenidos con la aplicación de la guía.

A partir de los resultados de la aplicación guía, se puede establecer que las estrategias de moldeamiento y el uso de juego de roles favorecen el desarrollo adecuado de las habilidades sociales abordadas. Lo anterior, en vista de que estas estrategias fueron mejor, permitieron que los participantes lograran desarrollar o fortalecer los repertorios de manera apropiada, pues se identifica la ejecución érronea o por debajo del rendimiento esperado y se corrige mediante el refuerzo de conductas moleculares pertenecientes a las habilidades en específico. Asimismo, se logró identificar, para el desarrollo de habilidades sociales con mayor complejidad, habilidades para hacer frente al estrés y habilidades alternativas a la agresión. Admás, se considera útil tener en cuenta conductas moleculares pertenecientes a las habilidades sociales avanzadas o básicas, aspecto abordado más adelante.

Por otra parte, se reconoce que, en ocasiones, la aplicación de las habilidades de forma adecuada en actividades lúdicas fue reducida, por lo que se considera relevante mejorar dichas actividades, con la finalidad de que se pueda apreciar con mayor efectividad la adquisición de las habilidades sociales adecuadas. Igualmente, se evidenció escasa aplicación de las tareas dejadas sesión tras sesión, de allí que se sugiera buscar estrategias que favorezcan la realización de las tareas por parte del grupo participante, en vista de que este tipo de estrategias contribuyen a la consolidación de los repertorios conductuales relacionados con habilidades sociales en la cotidianidad de los participantes.

Así mismo, la literatura reporta que estrategias como el modelamiento, tanto de los psicólogos como de los pares, las instrucciones verbales (y dar explicaciones claras y precisas), los juegos de roles (imitar las conductas a entrenar con base en las historias incluidas en las guías), la retroalimentación, señalando a cada participante aquello que había realizado de forma correcta y las oportunidades de mejora, y el reforzamiento positivo 
mediante elogios, además del tiempo de juego libre, entre otras, posibilitan el incremento de las conductas objetivo, en este caso la puesta en práctica de las diferentes habilidades sociales (González et al., 2012), lo cual se evidencia en los resultados de la guía empleada. Igualmente, los resultados de la presente investigación permiten asumir que, como proponen Caballo et al. (2015) y Vagos et al. (2015), el contexto escolar puede constituir un entorno adecuado para la realización de intervenciones en habilidades sociales.

Por otra parte, se evidenció el aumento de los repertorios de habilidades sociales adecuados durante el transcurso de las sesiones cumpliendo con el orden establecido de las habilidades propuestas por Goldstein et al. (1989). Es necesario tener en cuenta que el abordaje cognitivo-conductual establece que el trabajo de las habilidades sociales requiere dos grados de análisis: uno molecular y otro molar; lo que implica que para que se configuren aspectos complejos de las habilidades sociales, deben desarrollarse primero conductas muy específicas de contacto social básico (Caballo, 1993). La aplicación de la guía como está planteada permitió que el aprendizaje de las habilidades sociales fuera progresivo, empezando desde las habilidades básicas hasta las habilidades de planificación. Investigaciones como la realizada por Morales, Benítez y Agustín (2013), sugieren que la aproximación sucesiva de conductas y el cumplimiento del orden de la intervención de habilidades sociales, permite una mejora de manera progresiva, consolidando el aprendizaje a través de las sesiones, aspecto que se puede apreciar con relación a los resultados obtenidos.
Ahora bien, es importante resaltar que, durante las sesiones se evidenció resistencia por parte de los adolescentes participantes a temas relacionados con las emociones, especialmente en las sesiones de entrenamiento de habilidades relacionadas con los sentimientos. Este factor puede estar influido por el periodo del ciclo vital, debido a que es habitual que durante la adolescencia existan limitaciones en el reconocimiento emocional (Calvo et al., 2014). De la misma manera, Márquez y Gaeta (2017) aportan que, al momento de impartir temas relacionados con las emociones, se considera necesario realizar una explicación rigurosa y profunda sobre la expresión de sentimientos, teniendo en cuenta el conocimiento preexistente y las posibles impresiones erróneas a nivel social alrededor de esta temática. Por lo anterior, se considera importante abordar el área emocional de forma constante durante los ejercicios de intervención, al igual que resulta recomendable tener en cuenta la compañía del colegio y la familia en el proceso, esto con el fin de trabajar sobre los mismos objetivos y lograr un abordaje integral que beneficie a los adolescentes (Márquez \& Gaeta, 2017).

Otro factor a considerar es la cantidad de participantes en el grupo experimental. Cabe mencionar que las sesiones se realizaron con un grupo de 27 adolescentes, para lo cual, de acuerdo con Vidal y Fuertes (2014), se requiere de un facilitador o terapeuta con altos niveles de experticia en el manejo de grupos; ya que se entiende que los grupos pueden estar configurados por distintos tipos de subjetividades, que influyen en la realización de actividades en una intervención socioeducativa, lo que exige entrenamiento previo en técnicas para el trabajo grupal, 
en especial con niños y adolescentes, al igual que una relación cercana con los participantes de la intervención (Guerrero et al., 2017). Esta recomendación se origina a raíz de que los profesionales en formación fueron los ejecutores principales de la guía, y su experticia en el manejo de grupos era reducida.

En ese sentido, es importante el trabajo con un grupo más pequeño. Igualmente, esta sugerencia se realiza puesto que los grupos pequeños facilitan la interacción, confianza y cercanía entre los miembros de forma adecuada (Vidal \& Fuertes, 2013). Esto puede conllevar a la participación más activa por parte de los adolescentes y favorecer el cumplimiento de los objetivos de cada sesión.

Por otra parte, se identificó que resulta relevante la implementación de más actividades lúdicas que involucren el juego favoreciendo el proceso de relación terapéutica con los participantes. Cabe mencionar que dentro de la terapia psicológica el juego fomenta la comunicación e interacción social positiva, permitiendo desarrollar un relacionamiento adecuado en el grupo mediante la generación de un ambiente cálido, confortable y seguro que alienta a la expresión emocional adecuada (Schaefer, 2012).

Por otro lado, se considera útil incluir sesiones de seguimiento que permitan dar cuenta del mantenimiento de los resultados a largo plazo en el desarrollo de repertorios de habilidades sociales efectivas o disminución en conductas problemáticas como agresividad, bullying, bajo rendimiento académico, etc. Por último, resulta interesante implicar a los padres de familia en la intervención, pues es posible que su involucramiento contribuya a la presencia de resultados positivos en los participantes.

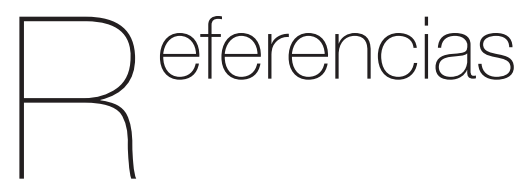

Albrecht, S. F., Mathur, S. R., Jones, R. E., \& Alazemi, S. (2015). A school-wide threetiered program of social skills intervention: Results of a three-year cohort study. Education and Treatment of Children, 38(4), 565-586.

Bautista, L., Plata, M., Hernández, L., Herrera, M. \& Parra, N. (2017). Adaptación y validación de instrumento para evaluar habilidades psicosociales y hábitos saludables en escolares. Universidad y Salud, 19(3), 366-377. http://dx.doi. org/10.22267/rus.171903.99.

Caballo, V. (1993). Manual de evaluación y entrenamiento en habilidades sociales. Madrid: Siglo XXI.

Caballo, V. E., Carrillo, G. B., \& Ollendick, T. H. (2015). Eficacia de un programa lúdico de entrenamiento en habilidades sociales para la intervención sobre la ansiedad social en niños. Psicología Conductual, 23(3), 403-427.

Calvo, R., Solórzano, G., Morales, C., Kassem, M., Codesal, R., Blanco, A., \& Gallego, L. (2014). Procesamiento emocional en pacientes TCA adultas vs. adolescentes. Reconocimiento y regulación emocional. Clínica y Salud, 25(1), 19-37. 
Díaz, C., Díaz, M. A., \& Frost, D. (2018). Habilidades Sociales en Adolescentes: Una Guía de Intervención Grupal. Material no publicado.

Gadaire, D. M., Marshall, G., \& Brissett, E. (2017). Differential reinforcement of low rate responding in social skills training. Learning and Motivation, 60, 34-40. https://doi. org/10.1016/j.Imot.2017.08.005.

Gaete, V. (2015). Desarrollo psicosocial del adolescente. Revista Chilena de Pediatría, 86(6), 436-443. https://dx.doi.org/10.1016/j. rchipe.2015.07.005.

Garaigordobil, M., \& Peña, A. (2014). Intervención en las habilidades sociales: efectos en la inteligencia emocional y la conducta social. Psicología Conductual, 22(3), 551-567.

García-Castellar, R., Jara-Jiménez, P., SánchezChiva, D., \& Mikami, A. Y. (2015). Social skills deficits in a virtual environment among spanish children with ADHD. Journal of Attention Disorders, 22(8), 776-786. https:// doi.org/10.1177/1087054715591850.

Goldstein, A., Sprafkin, R., Gershaw, J., \& Klein, P. (1989). Habilidades sociales en la adolescencia: un programa de enseñanza. Barcelona: Ediciones Martínez Roca.

González, C., Ampudia, A., \& Guevara, Y. (2012). Programa de intervención para el desarrollo de habilidades sociales en niños institucionalizados. Acta colombiana de Psicología, 15(2), 43-52.

Gur, C. (2015). Self-knowledge and Adolescence. Procedia-Social and Behavioral Sciences, 197, 1716-1720.
Guerrero, P., Aguirre, C., Besser, C., Morales, M., Salinas, J., \& Zamora, M. (2017). Talleres socioeducativos en infancia desde un enfoque situado. Revista Latinoamericana de Ciencias Sociales, Niñez y Juventud, 15(1), 249-265.

Harrell, A. W., Mercer, S. H., \& DeRosier, M. E. (2009). Improving the socialbehavioral adjustment of adolescents: The effectiveness of a social skills group intervention. Journal of Child and Family Studies, 18(4), 378-387.

Hernández, R., Fernández, C. \& Baptista, M. D. (2014). Metodología de la investigación. México D.F.: Mc Graw Hill.

Holopainen, L., Lappalainen, K., Junttila, N., \& Savolainen, H. (2012). The role of social competence in the psychological well-being of adolescents in secondary education. Scandinavian Journal of Educational Research, 56(2), 199-212.

Instituto Colombiano de Medicina Legal y Ciencias forenses (2018). Forensis 2017. Datos para la vida. Recuperado de http:// www.medicinalegal.gov.co/documents/ 20143/262076/Forensis+2017+Interactivo. pdf/ 0 a 09 fedb-f 5 e 8 - 11 f 8 - 71 ed 2d3b475e9b 82

Korem, A., Horenczyk, G. \& Tatar, M. (2012). Inter-group and intra-group assertiveness: Adolescents' social skills following cultural transition. Journal of Adolescence, 35(4), 855-862.

Li, Y., Coplan, R. J., Wang, Y., Yin, J., Zhu, J., Gao, Z. \& Li, L. (2016). Preliminary evaluation of a social skills training and facilitated play early intervention programme for extremely shy young children in China. Infant and Child Development, 25(6), 565-574. 
López de Mesa, C., Carvajal, C. A., Soto, M. F., \& Urrea, P. N. (2013). Factores asociados a la convivencia escolar en adolescentes. Educación y Educadores, 16(3), 383-410. Recuperado de http://www.scielo.org.co/ pdf/eded/v16n3/v16n3a01.pdf.

Mafra, H. (2015). Development of Learning and Social Skills in Children with Learning Disabilities: An Educational Intervention Program. Procedia - Social and Behavioral Sciences, 209, 221-228. https://doi. org/10.1016/J.SBSPRO.2015.11.220.

Márquez, M., \& Gaeta, M. (2017). Desarrollo de competencias emocionales en pre-adolescentes: el papel de padres y docentes. Revista Electrónica Interuniversitaria de Formación del Profesorado, 20(2), 221-235.

McDaniel, S. C., Bruhn, A. L., \& Troughton, L. (2017). A brief social skills intervention to reduce challenging classroom behavior. Journal of Behavioral Education, 26(1), 53-74.

Mendo, S., León del Barco, B., Castaño, E. F., \& Polo del Rio, M. I. (2016). Entrenamiento en habilidades sociales en el contexto universitario: efecto sobre las habilidades sociales para trabajar en equipos y la ansiedad social. Psicología Conductual, 24(3), 423-438.

Morales, M., Benítez, M. \& Agustín, D. (2013). Habilidades para la vida (cognitivas y sociales) en adolescentes de una zona rural. Revista Electrónica de Investigación Educativa, 15(3), 98-113

Morán, V. E. \& Olaz, F. O. (2014). Instrumentos de evaluación de habilidades sociales en América Latina: un análisis bibliométrico.
Revista de Psicología, 23(1), 93-105. doi: 10.5354/0719-0581.2014.328770

Patrício do Amaral, M., Maia, F.J, \& Bezerra de Medeiros, C.R. (2015). Las habilidades sociales y el comportamiento infractor en la adolescencia. Subjetividad y procesos cognitivos, 19(2), 17-38.

Pedrosa, l., Juarros-Basterretxea, J., RoblesFernández, A., Basteiro C, J. \& GarcíaCueto, E. (2015). Pruebas de bondad de ajuste en distribuciones simétricas, ¿qué estadístico utilizar?. Universitas Psychologica, 14(1), 15-24.

Probst, T., Geib, C., Güroff, E., \& Mühlberger, A. (2017). Training the social skill "being able to demand" vs. training the social skill "being able to say no". A randomized controlled trial with healthy individuals. Journal of Behavior Therapy and Experimental Psychiatry, 57, 1-5. https://doi.org/10.1016/j. jbtep.2017.01.006.

Radley, K. C., Jenson, W. R., Clark, E., Hood, J. A., \& Nicholas, P. (2014). Using a multimedia social skills intervention to increase social engagement of young children with Autism Spectrum Disorder. Intervention in School and Clinic, 50(1), 22-28. https://doi. org/10.1177/1053451214532350.

Reyes, M.E. (2016). Relación entre Habilidades Sociales y Desempeño Docente desde la percepción de estudiantes adultos de universidad privada en Lima, Perú. Revista Digital de Investigación en Docencia Universitaria, 10(2), 17-31. https://dx.doi. org/10.19083/ridu.10.465.

Rodríguez, C., Gutiérrez, J. \& Pozo, T. (2014). Fundamentos conceptuales de las principales pruebas de significación estadística en el 
ámbito educativo. Recuperado de https://www.ugr.es/ erivera/ PaginaDocencia/Posgrado/Documentos/ ClementeCuadernoInferencial.pdf

Sanz, M. (2016). Terapia grupal: Manual para la acción. Madrid: Editorial 5.

Seema, G. B., \& Kumar, G. V. (2018). Impact of social skills training on self-esteem among male and female adolescent students. Indian Journal of Positive Psychology, 9(1), 147-151.

Schaefer, C.E (2012). Fundamentos de terapia de juego. México: Manual Moderno.

Vagos, P., Pereira, A., \& Warner, C. M. (2015). Effectiveness of skills for academic and social success (SASS) with Portuguese adolescents. International Journal of Group Psychotherapy, 65(1), 135-147.

Van der Stouwe, T., Asscher, J. J., Hoeve, M., van der Laan, P. H., \& Stams, G. J. J. (2018).
Social skills training (SST) effects on social information processing skills in justiceinvolved adolescents: Affective empathy as predictor or moderator. Children and Youth Services Review, 90, 1-7.

Van Vugt, E. S., Deković, M., Prinzie, P., Stams, G. J. J. M., \& Asscher, J. J. (2013). Evaluation of a group-based social skills training for children with problem behavior. Children and Youth Services Review, 35(1), 162-167.

Vidal, S., Fuertes, M. (2013). La dinámica de grupos para el trabajo cooperativo facilita la comunicación. Vivat Academia, (123), 1-12.

Wolstencroft, J., Robinson, L., Srinivasan, R., Kerry, E., Mandy, W., \& Skuse, D. (2018). A Systematic Review of Group Social Skills Interventions, and Metaanalysis of Outcomes, for Children with High Functioning ASD. Journal of Autism and Developmental Disorders, 48(7), 2293-2307. 